\title{
AS ESPÉCIES DE COPEPODA (CRUSTACEA: ERGASILIDAE) PARASITAS DOS FILAMENTOS BRANQUIAIS DE Potamorhina latior (Spix \& Agassiz, 1829) (CHARACIFORMES: CURIMATIDAE) DE LAGOS DE VÁRZEA DA AMAZÔNIA CENTRAL, BRASIL
}

\author{
Germán Augusto Murrieta MOREY ${ }^{1}$, Adria da Costa MOREIRA ${ }^{1}$, José Celso de Oliveira MALTA ${ }^{1}$ \\ 1 Instituto Nacional de Pesquisas da Amazônia, Laboratório de Parasitologia de Peixes Av. André Araújo 2936, Petrópolis, \\ CEP-69.067-375, Manaus, Amazonas, Brasil, Email: germantiss@hotmail.com
}

\section{RESUMO}

Três espécies de Copepoda foram coletadas nas brânquias de Potamorhina latior de seis lagos de várzea, cinco do rio Solimões e um do rio Purus, no estado do Amazonas: Amplexibranchius bryconis; Ergasilus jaraquensis e Miracetyma etimaruya. Potamorhina latior é um novo hospedeiro para A. bryconis e E. jaraquensis e o segundo registro para M. etimaruya. Amplexibranchius bryconis foi à espécie dominante (77\%) e com os maiores índices parasitários. As maiores prevalências ocorreram na vazante. Houve correlação negativa não significativa $(p>0,05)$ entre o comprimento de $P$. latior e a abundância. Foi ampliada a distribuição geográfica de $M$. etimaruya para o rio Solimões.

PALAVRAS-CHAVE: parasitos de peixes; Amplexibranchius; Ergasilus; Miracetyma; rio Solimões.

\section{THE SPECIES OF COPEPODA (CRUSTACEA: ERGASILIDAE) PARASITES OF GILL FILAMENTS OF Potamorhina latior (Spix \& Agassiz, 1829) (CHARACIFORMES: CURIMATIDAE) OF CENTRAL AMAZON FLOODPLAIN LAKES, BRAZIL}

\begin{abstract}
Three species of Copepoda were collected in the gills of Potamorhina latior from six floodplain lakes, five from Solimões River and one from the Purus River, Amazonas State: Amplexibranchius bryconis; Ergasilus jaraquensis and Miracetyma etimaruya. Potamorhina latior is a new host for A. bryconis and E. jaraquensis and the second record for M. etimaruya. Amplexibranchius bryconis was the dominant species $(77 \%)$ and with higher rates of parasites. The highest prevalence occurred in the dry season. There was no significant $(\mathrm{p}>0.05)$ negative correlation between the length of $P$. latior and abundance. There was expanded geographical distribution of M. etimaruya to the Solimões River.
\end{abstract}

KEYWORDS: fish parasites Amplexibranchius; Ergasilus; Miracetyma; Solimões River. 


\section{INTRODUÇÃO}

Potamorhina latior (Spix \& Agassiz, 1829) é um peixe da ordem Characiformes, família Curimatidae e ocorre na América do Sul na bacia do rio Amazonas. É uma espécie de porte médio e pode atingir até $30 \mathrm{~cm}$ de comprimento total. Tem hábito alimentar detritívoro, faz migrações tróficas e reprodutivas, desova no início da enchente, e habita lagos de água branca (Vari 1984, 2003).

As espécies de Copepoda são os crustáceos aquáticos que em termos de tamanho, diversidade $\mathrm{e}$ abundância podem ser vistos como os insetos dos mares. Mais de 10.000 espécies são conhecidas. Mas, sobre os harpacticoides bentônicos, os ergasilídeos, os sifonostomatoides associados aos invertebrados marinhos, pouco se conhece (Huys \& Boxshall, 1991; Varella \& Malta, 1998).

O conhecimento da fauna de crustáceos da família Ergasilidae nas Américas é irregular. Em algumas áreas geográficas eles têm sido estudadas intensamente por muitos anos. A fauna de ergasilídeos da América do Norte e a do Brasil na América Sul são as que têm o melhor conhecimento nas América (Suárez-Morales et al., 2008).

Na Amazônia são conhecidas 47 espécies de Copepoda parasitas de peixes. Trinta e cinco são da família Ergasilidae e incluídas em oito gêneros: 16 espécies de Ergasilus Nordmann, 1832; dez de Acusicola Cressey, 1970 (Amado \& Rocha, 1996); cinco de Brasergasilus Thatcher \& Boeger, 1983; uma de Amplexibranchius Thatcher \& Paredes, 1985; uma de Rhinergasilus Boeger \& Thatcher, 1988; duas de Prehendorastrus Boeger \& Thatcher, 1990; três de Miracetyma Malta, 1993 e uma de Pindapixara Malta, 1995 (Malta, 1993a; 1993b; Varella \& Malta, 1998; 2009).

Nas espécies de Copepoda parasitas dos peixes amazônicos, a característica mais importante, que as distinguem dos seus parentes de vida livre é a antena preênsil. Machos e fêmeas possuem antenas semelhantes, mas as dos machos são menos que a metade do tamanho das antenas das fêmeas Outro apêndice que mostra adaptações estruturais a um modo de vida parasitária é a primeira perna. Esta é morfologicamente diferente das demais com adaptação para a função alimentar levando o tecido do hospedeiro até a boca (Varella, 1985; Malta, 1993a; Varella \& Malta, 1998; 2009).

O objetivo do presente estúdio foi avaliar a composição de espécies de Copepoda (Crustacea: Ergasilidae) parasitas dos filamentos branquiais de Potamorhina latior de lagos de várzea da Amazônia central

\section{MATERIAL E MÉTODOS}

Os exemplares de $P$. latior foram capturados em seis lagos de várzea, cinco do rio Solimões: Baixio $\left(03^{\circ} 17^{\prime} 27,2^{\prime \prime} \mathrm{S} / 60^{\circ} 04^{\prime} 29,6^{\prime \prime} \mathrm{O}\right)$ no município de Iranduba; Preto $\left(03^{\circ} 21^{\prime} 17,1^{\prime \prime} \mathrm{S} / 60^{\circ} 37^{\prime} 28,6^{\prime \prime} \mathrm{O}\right)$ em Iranduba; Ananá $\left(03^{\circ} 53^{\prime} 54,8^{\prime \prime} \mathrm{S} / 61^{\circ} 40^{\prime} 18,4^{\prime \prime} \mathrm{O}\right)$ em Anori; Araçá (S03 $\left.{ }^{\circ} 45^{\prime} 04,3^{\prime \prime} \mathrm{S} / 62^{\circ} 21^{\prime} 25,9^{\prime \prime} \mathrm{O}\right)$ em Codajás; Maracá $\left(03^{\circ} 50^{\prime} 32,8^{\prime \prime S} / 62^{\circ} 34^{\prime} 32,4^{\prime \prime O}\right)$ em Coari e um lago do rio Purus, São Tomé $\left(03^{\circ} 49^{\prime}\right.$ $39,0^{\prime \prime} \mathrm{S} / 61^{\circ} 25^{\prime} 24,6^{\prime \prime} \mathrm{O}$ ), durante o período de fevereiro de 2012 a dezembro de 2013.

Foram realizadas oito expedições, quatro por ano durante as estações de cheia, vazante, seca e enchente. Os peixes foram capturados com redes de espera de $20 \mathrm{~m}$ de comprimento por $2 \mathrm{~m}$ de altura com malhas variando de 30, 40, 50, 60, 70, 80, 90 e $100 \mathrm{~mm}$ entre nós adjacentes. As redes ficaram na água 10 horas em cada lago no período diurno, com duas despescas.

No campo os peixes foram identificados, pesados e medidos. As brânquias foram removidas e preservadas em frascos de vidro preenchidos com água aquecida a $70^{\circ} \mathrm{C}$ e formol $4 \%$. Os frascos com as amostras de cada peixe foram rotulados com as informações correspondentes.

As brânquias foram analisadas no Laboratório de Parasitologia de Peixes do Instituto de Pesquisas da Amazônia (LPP - INPA) em Manaus. Sob microscópio estereoscópio os copépodos foram retirados dos filamentos branquiais, e conservados em álcool 70\%. Para o estudo morfológico das espécies de Copepoda, lâminas permanentes foram preparadas segundo o método de Eosina-Orange-G.

A identificação das espécies de Copepoda foi baseada nas características morfológicas e foram utilizados os trabalhos de Thatcher e Robertson (1982); Thatcher \& Paredes (1985) e Malta (1993a; 1993b).

Os descritores ecológicos: intensidade média; abundância média e prevalência foram calculados de acordo com Bush et al. (1997). O status comunitário das infracomunidades parasitárias foi classificado segundo Bush \& Holmes (1986): espécies centrais (presentes em mais de dois terços dos hospedeiros), espécies secundárias (em um a dois terços do hospedeiro) e espécies satélites (em menos de um terço do hospedeiro).

Foi calculado o Coeficiente de Dominância (CD). Este coeficiente mede a porcentagem de uma espécie em relação ao conjunto da infracomunidade parasitária para todos os hospedeiros examinados (Serra-Freire, 2002). O coeficiente de correlação de Pearson $r$ foi calculado para determinar possíveis correlações entre a abundância parasitária e o comprimento dos hospedeiros. Todos os valores foram considerados significativos quando $\mathrm{p}<0,05$. 


\section{RESULTADOS}

Cem exemplares de P. latior de seis lagos de várzea da Amazônia central foram capturados e examinados. Foram coletados 133 espécimens de Copepoda, 103 de Amplexibranchius bryconis Thatcher \& Paredes, 1985; 25 de Miracetyma etimaruya Malta, 1993 e cinco de Ergasilus jaraquensis Thatcher \& Robertson, 1982. A prevalência total foi de 26 e a intensidade variou de um a seis copépodes por peixe.

Amplexibranchius bryconis foi a espécie que apresentou os maiores índices parasitários e o maior número de indivíduos 103 . Os segundos maiores índices e números de indivíduos foram os de $M$. etimaruya. E, os menores foram de E. jaraquensis (Tabela 1). Amplexibranchius bryconis foi a maior das três espécies com a média de 819 micrômetros seguida de $E$. jaraquensis com 576. A menor foi $M$. etimaruya com 555. (Tabela 2).

Amplexibranchius bryconis e M. etimaruya ocorreram parasitando $P$. latior nas estações de vazante e seca e $E$. jaraquensis nas estações de cheia e vazante (Tabela 3). O status comunitário das infracomunidades de Copepoda de $P$. latior foi de espécies satélites (prevalência $<33 \%$ ). O coeficiente de correlação de Pearson $r$ teve uma correlação negativa não significativa entre o comprimento das P. latior e a abundância parasitaria $(r=-0,3891 ; p=$ 0,0545).

Tabela 1. Índices parasitários das espécies de Copepoda parasitas de Potamorhina latior (Spix \& Agassiz, 1829) capturados em lagos de várzea, do rio Solimões na Amazônia Central. P (\%) = Prevalência; NI = Número de indivíduos; IM = Intensidade media; $\mathrm{AM}$ = abundância média; $\mathrm{CD}=$ Coeficiente de dominância.

\begin{tabular}{lccccc}
\hline \multicolumn{1}{c}{ Espécie } & P (\%) & NI & IM & AM & CD \% \\
\hline Amplexibranchius bryconis & 16 & 103 & $6,44(1-51)$ & 1,03 & 77 \\
Miracetyma etimaruya & 16 & 25 & $1,56(1-4)$ & 0,25 & 19 \\
Ergasilus jaraquensis & 4 & 5 & $1,25(1)$ & 0,05 & 4 \\
\hline
\end{tabular}

Tabela 2. Medidas do corpo sem as setas caudais das três espécies de Copepoda parasita de Potamorhina latior (Spix \& Agassiz, 1829) de lagos de várzea, do rio Solimões na Amazônia Central.

\begin{tabular}{llc}
\hline \multicolumn{1}{c}{ Espécie } & Comprimento & Largura \\
\hline Amplexibranchius bryconis & $819(720-878)$ & $246(202-252)$ \\
Miracetyma etimaruya & $555(490-720)$ & $192(140-304)$ \\
Ergasilus jaraquensis & $576(538-602)$ & $201(193-210)$ \\
\hline
\end{tabular}


Tabela 3. Índices parasitários das três espécies de Copepoda parasitas de Potamorhina latior (Spix \& Agassiz, 1829) capturadas em lagos de várzea do rio Solimões durante as estações de enchente, cheia, vazante e seca. PP = peixes parasitados; $\mathrm{PE}=$ peixes examinados; $\mathrm{P} \%=$ prevalência $\mathrm{NI}=$ Número de indivíduos; $\mathrm{IM}=$ intensidade média; $A M=$ abundância média.

\begin{tabular}{llccccc}
\hline \multirow{2}{*}{ Parasita } & Estação & PE/PP & P\% & NI & IM & AM \\
\hline Amplexibranchius bryconis & Enchente & $7 / 0$ & - & - & - & - \\
& Cheia & $32 / 0$ & - & - & - & - \\
& Vazante & $31 / 11$ & 35,48 & 79 & $7,18 \pm 12,32$ & 2,55 \\
& Seca & $30 / a b r$ & 13,33 & 17 & $4,25 \pm 2,32$ & 0,57 \\
Miracetyma etimaruya & Enchente & $7 / 0$ & - & - & - & - \\
& Cheia & $32 / 0$ & - & - & - & - \\
& Vazante & $31 /$ dez & 38,71 & 17 & $1,42 \pm 0,87$ & 0,55 \\
& Seca & $30 /$ mar & 10 & 7 & $2,33 \pm 1,60$ & 0,10 \\
\hline \multirow{2}{*}{ Ergasilus jaraquensis } & Enchente & $7 / 0$ & - & - & - & - \\
& Cheia & $32 / 2$ & 6,25 & 3 & $1,5 \pm 0,71$ & 0,09 \\
& Vazante & $31 / 2$ & 6,45 & 2 & $01 \pm 0,33$ & 0,06 \\
\hline
\end{tabular}

\section{DISCUSSÃO}

Miracetyma etimaruya foi descrita dos filamentos branquiais de Curimata cyprinoides (Linnaeus, 1758) capturadas no rio Jamari próximo à Ariquemes. Também foi coletada de $C$. cyprinoides dos rios Jiparaná próximo à Jiparaná, Guaporé e Mamoré próximos à Surpresa. Miracetyma etimaruya foram coletadas também em Psectrogaster essequibensis (Gunther, 1864) do rio Pacaás-Novos próximo à Guajará-Mirim, todas localidades no estado de Rondônia (Malta, 1993a).

Posteriormente $M$. etimaruya foi coletada dos filamentos branquiais de Curimatella lepidura Eigenmann \& Eigenmann 1899 capturadas na represa de Três Marias no alto rio São Francisco, estado de Minas Gerais (Albuquerque et al., 2008). Neste trabalho é feito o primeiro registro de ocorrência de $M$. etimaruya parasitando $P$. latior capturadas no rio Solimões, no estado do Amazonas. Neste trabalho é ampliada a distribuição geográfica de $M$. etimaruya e os dados corroboram que existe uma alta especificidade parasitária entre $M$. etimaruya es peixes da família Curimatidae.

Amplexibranchius bryconis foi descrito dos filamentos branquiais de Brycon cephalus (sinonímia de B. amazonicus Spix \& Agassiz, 1829) da cidade de Iquitos no Peru (Thatcher \& Paredes 1985). Neste trabalho é feito o segundo registro de ocorrência de $A$. bryconis, o primeiro registro para o Brasil e um novo hospedeiro é conhecido, P. latior.
Ergasilus jaraquensis Thatcher \& Robertson, 1982 foi descrito dos filamentos branquiais de Semaprochilodus insignis (Jardine, 1841) do rio Solimões próximo a cidade de Manaus (Thatcher \& Robertson, 1982). Neste trabalho $P$. latior é registrada como um novo hospedeiro para $E$. jaraquensis.

A parasitofauna de Pygocentrus nattereri (Kner, 1858) capturadas em lagos de várzea do rio Solimões apresentou as maiores prevalências durante os meses de vazante e seca (Vital et al., 2011; Morais 2011). Neste trabalho as maiores prevalências das três espécies de Copepoda ocorreram nos meses da vazante.

Os padrões de correlação entre o comprimento do peixe e a riqueza, a intensidade e a abundância de parasitos têm correlações positivas fracas não significativas ou mesmo correlações negativas (Saad-Farees \& Combes 1992). Isto ocorre devido à regulação dependente de intensidade (limitação de tamanho e conteúdo de nutrientes). Essa regulação é mais intensa em peixes de maior tamanho do que nos menores (Poulin \& Morand, 1999).

Os peixes são considerados "ilhas" disponíveis para colonização por parasitas (Gravel et al., 2011; Poulin 2014). As ilhas de maior tamanho, idade e com grande distribuição geográfica abrigam uma maior riqueza e abundância de espécies por um processo de acumulação dos parasitos (Poulin, 2007; 2014). 
A influência do tamanho do peixe sobre a riqueza e abundância das espécies parasitas e a aplicação da teoria de biogeografia de ilhas é um assunto bastante controverso (Higgs 1981; Albuquerque 2009). Porque o acúmulo de parasitos em peixes de maior tamanho é evitado pela regulação dependente de intensidade (Poulin, 2007; Bellay et al., 2013). Por isso são comuns correlações positivas ou negativas não significativas (Saad-Farees e Combes 1992).

Ectoparasitos de monoxenos podem ter a relação com o tamanho do hospedeiro influenciada pelo grau de especialização dos órgãos de fixação e pela disponibilidade das formas infectantes a determinadas faixas da população de hospedeiros (Tavares et al., 2001). Algumas espécies de parasitas, em função de algumas peculiaridades morfológicas, seu ciclo biológico e local de infestação, podem apresentar um padrão uniforme de distribuição (Luque et al., 1996).

As correlações são influenciadas por restrições biológicas (fisiologia e imunologia) e ecológicas (dinâmica de distribuição) das populações hospedeiras. Também por eventos estocásticos e/ou interações bióticas como competição, regulação dependente de intensidade, inimigos naturais e dieta. Tais restrições evitam altas intensidades, abundância e riqueza de espécies parasitas nos peixes (Alves \& Luque 2006).

Neste trabalho houve correlação negativa não significativa entre o comprimento de $P$. latior e a abundância das espécies parasitarias. Esse tipo de correlação pode ser atribuído às estratégias do ciclo de vida de cada espécie, ao grau de especialização dos órgãos de fixação do parasita e a disponibilidade de formas livres infetantes a determinadas faixas etárias da população hospedeira.

\section{AGRADECIMENTOS}

Agradecemos ao Projeto PIATAM e ao Instituto Nacional de Pesquisas da Amazônia (INPA) pelo apoio logístico, e a toda equipe do Laboratório de Parasitologia e Patologia de Peixes pelo auxílio técnico dado durante o estudo.

\section{BIBLIOGRAFIA CITADA}

Albuquerque, M.C. 2009. Taxonomia e aspectos ecológicos da fauna parasitária de Triportheus guentheri (Garman, 1890) e Tetragonopterus chalceus Spix \& Agassiz, 1829 do Reservatório de Três Marias, Alto Rio São Francisco, Minas Gerais, Brasil. Dissertação de Mestrado, Universidade Federal Rural do Rio de Janeiro, Seropédica. $106 \mathrm{pp}$.
Albuquerque, M.C.; Osório, A.M.; Thatcher, V.E.; Brasil-Sato, M.C. 2008. Copepod parasites of Curimatella lepidura (Characiformes, Curimatidae) from the Três Marias Reservoir, Brasil. Arquivos Brasileiros de Medicina Veterinária e Zootecnia, 60(5): 1271-1273.

Alves, D.R.; Luque, J.L. 2006. Ecologia das comunidades de metazoários parasitos de cinco espécies de escombrídeos (Perciformes: Scombridae) do litoral do estado do Rio de Janeiro, Brasil. Revista Brasileira de Parasitologia Veterinária, 15(4): 167-181.

Bush, A.O.; Holmes, J.C. 1986. Intestinal helmints of lesser scaup ducks: an interactive community. Canadian Journal Zoology, 64: 142-152.

Bush, A.O.; Lafferty, K.D.; Lotz, J.M.; Shostak, A.W. 1997. Parasitology meets ecology on its own terms. Journal of Parasitology, 83: 575583.

Gravel, D.; Massol, F; Canard, E.; Mouillot, D.; Mouquet, N. 2011. Trophic theory of Island Biogeography. Ecology Letters, 14: 1010-1016.

Higgs, A.J. 1981. Island biogeography theory and nature reserve design. Journal of Biogeography, 8(2): 117-124.

Huys, R.; Boxshall, G.A. 1991 Copepod evolution. The Ray Society, London, UK. 468 pp.

Luque, J.L.; Amato, J.F.R.; Takemoto, R.M. 1996. Comparative analysis of the communities of metazoan parasites of Orthopristis ruber and Haemulon steindachneri (Osteichthyes: Haemulidae) from the southeastern Brazilian litoral: I. structure and influence of the size and sex of hosts. Revista Brasileira de Biologia, 56(2): 279-292.

Malta, J.C.O. 1993a. Miracetyma etimaruya gen. et sp. n. (Copepoda, Poecilostomatoida, Ergasilidae) from freshwater fishes of the Brazilian Amazon. Acta Amazonica, 23(1): 4957.

Malta, J.C.O. 1993b. Brasergasilus guaporensis sp. n. (Copepoda, Ergasilidae) das brânquias de Leporinus fasciatus (Bloch, 1890) (Characiformes: Anostomidae) da Amazônia brasileira. Acta Amazonica, 23(4): 441-447.

Malta, J.C.O.; Varella, A. 1996. Ergasilus turucuyus sp. n. (Copepoda: Ergasilidae) das brânquias de Acestrorhynchus falcatus (Block, 1794) e $A$. falcirostris (Cuvier, 1819), (Characiformes: Characidae) da Amazônia brasileira. Acta Amazonica, 26(1/2): 69-76. 
Morais, A.M. 2011. Biodiversidade de parasitos da piranha vermelha Pygocentrus nattereri (Kner, 1858) (Characiformes; Serrasalmidae) e sua avaliação como bioindicadores na Amazônia Central. Tese de doutorado. INPA, Manaus, 243 pp.

Poulin, R. 2007. Are there general laws in parasite ecology? Parasitology, 134 (6): 763-776.

Poulin, R. 2014. Parasite biodiversity revisited: frontiers and constraints. International Journal of Parasitology, 20: 1-9.

Poulin, R.; Morand, S. 1999. Geographical distances and the similarity among parasite communities of conspecific host populations. Parasitology, 119:369-374.

Suárez-Morales, E.; Santana-Piñeros, A.M. 2008. A new species of Ergasilus (Copepoda: Cyclopoida: Ergasilidae) from coastal fishes of the Mexican Pacific. Folia Parasitologica, 55: 224-230.

Tavares, L.E.R.; Luque, J.L.; Botelho Neto, S.L. 2001. Ecologia da comunidade de metazoários parasitos do olho-de-cão Priacanthus arenatus (Cuvier, 1829) (Osteichthyes, Priacanthidae) do litoral do estado do Rio de Janeiro, Brasil. Revista Brasileira de Zoociências, 3(1): 45-49.

Thatcher, V.E. 2006. Amazon Fish Parasites. In: Adis, J.; Arias, J.R.; Rueda-Delgado, G.; Wantzen, K.M. (Eds.). Aquatic Biodiversity in Latin America. 2nd edition, Pensoft Publishers, Praga, Bulgaria. 508 pp.

Thatcher, V.E.; Robertsom, B.A. 1982. The parasitic crustaceans of fishes from the Brazilian Amazon. 3. Ergasilus jaraquensis n. sp. (Copepoda: Cyclopoidea) from the gills of Semaprochilodus insignis (Schomburgk). Revista Brasileira de Biologia, 42(3): 515-519.

Thatcher, V.E.; Paredes, V. 1985. Amplexibranchius bryconis gen. et sp. nov. (Ergasilidae: Acusicolinae), from an Amazonian fish and remarks on the importance of leg morphology in this subfamily. Amazoniana, 9(2): 205-214.
Varella,A.M.B. 1985. O ciclo biológico de Ergasilus bryconis Thatcher, 1981 (Copepoda: Poecilostomatoida: Ergasilidae) parasita das brânquias do matrinxã, Brycon erythropterum (Cope, 1872) e aspectos de sua ecologia. Ed. Universitária, Natal, Brasil. 100 pp.

Varella, A.; Malta, J.C.O.1998. Maxillopoda Copepoda - Poecilostomatoida. p. 241 - 249. In: Yong, P.(Ed.). Catalogue of Crustacea of Brasil. Museu Nacional, Rio de Janeiro.

Varella, A.M.B.; Malta, J.C.O.; 2009. Copepoda Cyclopoida e Poecilostomatoida. p. 21-24. In: Fonseca, C.R.V.; Magalhães, C.; Rafael, J.A.; Franklin, E. (Eds.). A fauna de artrópodes da Reserva Florestal Adolpho Ducke. Estado Atual do Conhecimento Taxonômico e Biológico. Instituto Nacional de Pesquisas da Amazônia, Manaus, Amazonas.

Vari, R.P. 1984. Systematic of the Neotropical characiform genus Potamorhina (Pisces: Characiformes). Smithsonian Contribution for Zoology, 400: 1-36.

Vari, R.P. 2003. Family Curimatidae. p.51-64. In: Reis, R.E.; Kullander, S.O.; Ferraris, C.J., Jr. (Eds.) Check list of the freshwater fishes of South and Central America. Edipucrs, Porto Alegre, Brasil.

Vital, J.F.; Varella, A.M.B.; Porto, D.B.; Malta, J.C.O. 2011. Sazonalidade da fauna de metazoários de Pygocentrus nattereri (Kner, 1858) no lago Piranha (Amazonas, Brasil), e a avaliação de seu potencial como indicadora da saúde do ambiente. Biota Neotropica, 11(1): 16.

Recibido: 28 de setiembre del 2015

Aceptado para publicación: 11 de noviembre del 2015 